\title{
Sensitivity Analysis on Sea Surface Temperature Estimation Methods with Thermal Infrared Radiometer Data through Simulations
}

\author{
Kohei Arai ${ }^{1}$ \\ Graduate School of Science and Engineering \\ Saga University \\ Saga City, Japan
}

\begin{abstract}
Sensitivity analysis on Sea Surface Temperature: SST estimation with Thermal Infrared Radiometer: TIR data through simulations is conducted. Also Conjugate Gradient Method: CGM based SST estimation method is proposed. SST estimation error of the proposed CGM based method is compared to the conventional Split Window Method: SWM with a variety of conditions including atmospheric models. The results show that the proposed CGM based method is superior to the SWM.
\end{abstract}

Keywords-SST estimation; Split Window; Conjugate Gradient; MODTRAN; atmospheric model

\section{INTRODUCTION}

Sea Surface Temperature: SST estimation with thermal infrared radiometer onboard satellites is well known and widely used in a variety of research fields, in particular climate changes, global warming, etc. SST estimation methods are proposed [1]-[4]. Most of these are based on regressive analysis and use several spectral bands in Thermal Infrared: TIR wavelength region. The most dominant atmospheric factor is precipitable water. Using the different wavelength TIR bands whose influences due to water vapor are different, it is possible to reduce the influence. The most popular method is Multi Channel Sea Surface Temperature: MCSST [5]. Also previously proposed SST estimation methods are summarized by I. Barton [6]. In the same time, comparative study among the previously proposed methods is well reported [7].

Based on radiative transfer equation, inversion based SST estimation method is proposed [8]. Nonlinear radiative transfer equation is linearized then optimum combination of wavelength regions are selected [9]. Other than that, Geographic Information System: GIS based neural network is proposed for SST estimation method [10]. In this paper, sensitivity analysis results are described. SST estimation accuracy, in general, depends on relative humidity, air temperature, meteorological range, wind speed, aerosol type, and so on. Sensitivity of these factors on SST estimation accuracy is clarified in order to make clear that how does component influencing to SST estimation accuracy.

The following section describes the method for sensitivity analysis together with some theoretical background followed by some experiments. Then conclusion is described together with some discussions.

\section{PROPOSED METHOD}

A. Theoretical Background on SST Estimation with Thermal Infrared Radiometer Data

Radiation from a blackbody with physical temperature of $\mathrm{T}$ is expressed in equation (1)

$$
B_{\nu}(T)=\frac{2 h c^{2}}{\lambda^{5}\left(\exp \left(\frac{h c}{\lambda k T}\right)-1\right)}\left[W \cdot c m^{-2} \cdot s r^{-1} \cdot \mu m^{-1}\right]
$$

where

$$
\begin{aligned}
& \mathrm{k}: \text { Boltzman constant }[J / K] \\
& \mathrm{h}: \text { Plank constant }[J \cdot s] \\
& \mathrm{c}: \text { Light speed }[\mathrm{m} / \mathrm{s}] \\
& \lambda: \text { Wavelength at wave number } \nu
\end{aligned}
$$

The contribution from the atmosphere can be expressed as follows,

$$
\tau\left(\theta, z_{\infty}, z\right)=\exp \left\{-\int_{z}^{z_{\infty}} \frac{\rho(z) k(z)}{\cos (\theta)} d z\right\}
$$

where

$$
\begin{aligned}
& \theta: \text { Observation zenith angle } \\
& \rho: \text { Density of atmospheric continuents } \\
& k: \text { Volume extinction coefficient }
\end{aligned}
$$

and

$\int_{z}^{z_{\infty}} \frac{\rho(z) k(z)}{\cos (\theta)} d z$

is called as optical depth of the atmosphere.

\section{B. At Sensor Radiance of Thermal Infrared Radiometer}

For sea surface observation with TIR radiometers onboard remote sensing satellites, radiance includes three components, the contribution from sea surface, the contribution from the reflected radiance at sea surface and the contribution from the atmosphere. 


$$
\begin{array}{r}
I(\theta)=\int_{\lambda_{1}}^{\lambda_{2}} \Phi(\lambda)\left[\left\{\epsilon_{\lambda} B_{\lambda}\left(T_{s}\right)+\left(1-\epsilon_{\lambda}\right)\right.\right. \\
\left.\int_{z_{s}}^{\infty} B_{\lambda}[T(z)] \frac{\partial \tau\left(\theta, z_{\infty}, z\right)}{\partial z} d z\right\} \cdot \tau\left(\theta, z_{\infty}, z_{s}\right) \\
\left.\quad+\int_{z_{s}}^{\infty} B_{\lambda}[T(z)] \frac{\partial \tau\left(\theta, z_{\infty}, z\right)}{\partial z} d z\right] d \lambda
\end{array}
$$

where

$$
\begin{aligned}
T_{s} & : \text { Sea surface temperature }[\mathrm{K}] \\
\Phi & : \text { Spectral response function } \\
\epsilon & : \text { Emissivity } \\
\tau & : \text { Transparency }
\end{aligned}
$$

Spectral response function means spectral sensitivity function of spectral bands of TIR onboard satellites. In general, emissivity of sea surface in TIR wavelength region is almost 1 . Therefore, the second term of the equation (3) can be neglected.

$$
\begin{aligned}
I(\theta)= & \int_{\lambda_{1}}^{\lambda_{2}} \Phi(\lambda)\left\{\epsilon_{\lambda} B_{\lambda}\left(T_{s}\right) \tau\left(\theta, z_{\infty}, z_{s}\right)\right. \\
& \left.+\int_{z_{s}}^{\infty} B_{\lambda}[T(z)] \frac{\partial \tau_{\lambda}\left(\theta, z_{\infty}, z\right)}{\partial z} d z\right\} d \lambda
\end{aligned}
$$

\section{Method for Sensitivity Analysis}

By using MODTRAN of radiative transfer software code with six default atmospheric models, Tropic, Mid. Latitude Summer and Winter, Sub Arctic Summer and Winter, and 1976 US standard atmosphere, brightness temperature of the assumed spectral bands in Thermal Infrared wavelength regions can be estimated. Therefore Root Mean Square Error: RMSE of SST estimation error can be estimated for the assumed SST estimation method.

\section{Assumed SST Estimation Method}

Assuming spectral response function in the spectral wavelength region of spectral band is 1 , then the equation (4) and be rewritten as follows,

$$
\begin{aligned}
I(\theta) & =B_{\lambda}\left(T_{s}\right) \tau\left(\theta, z_{\infty}, z_{s}\right) . \\
& +\int_{z_{s}}^{\infty} B_{\lambda}[T(z)] \frac{\partial \tau_{\lambda}\left(\theta, z_{\infty}, z\right)}{\partial z} d z
\end{aligned}
$$

The second term of equation (5) can be approximated as follows,

$$
\int_{z_{s}}^{\infty} B_{i}[T(z)] \frac{\partial \tau_{i}\left(\theta, z_{\infty}, z\right)}{\partial z} d z=\left[1-\tau_{i}\left(\theta, z_{\infty}, z\right)\right] I_{a i}
$$

Where $I_{a i}$ denotes representative of spectral band $i$ of radiance. Atmospheric transparency can be rewritten as follows,

$$
\begin{gathered}
\tau_{i}(u, \theta)=c_{1 i} \exp \left[-1\left(c_{2 i}+c_{3 i} m\right) u^{c_{4 i}+c_{5} m}\right] \\
=c_{1 i} \exp \left[-\left(c_{2 i}+\frac{c_{3 i}}{\cos \theta}\right) u^{c_{4 i}+\frac{c_{5} i}{\cos \theta}}\right] \\
m \approx 1 / \cos \theta
\end{gathered}
$$

where $u$ denotes perceptible water while $m$ denotes slant length between sea surface and TIR instrument onboard satellites. In the TIR wavelength region, perceptible water is major absorbing continuants in the atmosphere. Through simulation studies with radiative transfer code of MODTRAN with six atmospheric models (Tropic, Mid. Latitude Summer, Mid. Latitude Winter, Sub Arctic Summer, Sub Arctic Winter and 1976 US Standard Atmosphere), the coefficients are obtained as shown in Table 1. Then $I_{a i}$ is calculated as follows,

$$
I_{a i}=F_{i}\left(I_{a k}\right)=A_{1 i}+A_{2 i} I_{a k}
$$

The coefficients in the equation (8) are calculated with MODTRAN in the same manner which is mentioned above. Table 2 shows the results.

TABLE I. COEFFICIENTS OF EQUATION (7) OBTAINED WITH MODTRAN OF ATMOSPHERIC SOFTWARE CODE

\begin{tabular}{|c||c|c|c|}
\hline & $c_{n 1}$ & $c_{n 2}$ & $c_{n 3}$ \\
\hline$c_{1 i}$ & 0.8507924 & 0.9356485 & 0.9253728 \\
\hline$c_{2 i}$ & -0.0754923 & -0.03505476 & -0.03752114 \\
\hline$c_{3 i}$ & 0.175898 & 0.08923810 & 0.1261287 \\
\hline$c_{4 i}$ & 1.451688 & 1.739096 & 1.679308 \\
\hline$c_{5 i}$ & -0.2339985 & -0.1563839 & -0.1293923 \\
\hline
\end{tabular}

TABLE II. COEFFICIENTS OF EQUATION (8) OBTAINED WITH MODTRAN OF ATMOSPHERIC SOFTWARE CODE

\begin{tabular}{|c||c|c|c|}
\hline & $A_{n 1}$ & $A_{n 2}$ & $A_{n 3}$ \\
\hline$A_{1 i}$ & $-0.88610 \times 10^{-6}$ & 0.0 & $0.75270 \times 10^{-6}$ \\
\hline$A_{2 i}$ & 0.62180 & 1.0 & 1.0590 \\
\hline
\end{tabular}

Consequently, radiance of spectral band $i$ can be expressed as follows,

$I_{i}=B_{i}\left[T_{s}\right] \tau_{i}(u, \theta)+\left[1-\tau_{i}(u, \theta)\right] F_{i}\left(I_{a k}\right)$

In order to avoid divergence of the solution, the following conditional equation is introduced.

$X=\frac{X_{\max }+X_{\min }}{2}+\frac{X_{\max }-X_{\min }}{\pi} \arctan \xi$

The unknown factors are as follows,

$\boldsymbol{x}=\left(T_{s}, u, I_{a k}\right)$

Namely, sea surface temperature, perceptible water, and representative radiance. The following cost function is introduced,

$J(\boldsymbol{x})=\sum_{i=1}^{3}\left(I_{i}-\hat{I}_{i}\right)^{2}$

Then iteration is stopped when the cost function is below the designated value,

$J(\boldsymbol{x}) \leq \varepsilon$

Radiance of the spectral band $i$ can be rewritten as follows, 


$$
\begin{aligned}
I_{i} & =\frac{c 1 c_{1 i}}{\lambda^{3} \exp \left(\frac{c 2}{\lambda T_{s}}\right)-1} \exp \left\{-\left(c_{2 i}+\frac{c 3 i}{\cos \theta}\right) u^{c_{4 i}+\frac{c 5 i}{\cos \theta}}\right\} \\
& +\left[1-c_{1 i} \exp \left\{-\left(c_{2 i}+\frac{c_{3 i}}{\cos \theta}\right) u^{c_{4 i}+\frac{c 5 i}{\cos \theta}}\right]\left(C_{1 i}+C_{2 i} I_{a k}\right)\right.
\end{aligned}
$$

Then the following updating equation is introduced,

$$
\boldsymbol{x}^{(n+1)}=\boldsymbol{x}^{(n)}+\beta^{(n)} A^{(n)} \nabla J\left[\boldsymbol{x}^{(x)}\right]
$$

It is rewritten in matrix and vector as follows,

$$
\begin{aligned}
& \left(\begin{array}{c}
T_{s} \\
u \\
I_{a k}
\end{array}\right)^{(n+1)}=\left(\begin{array}{c}
T_{s} \\
u \\
I_{a k}
\end{array}\right)^{(n)} \\
& +\beta^{(n)}\left(\begin{array}{ccc}
\frac{\partial^{2} J}{\partial T_{s}^{2}} & \frac{\partial^{2} J}{\partial T_{s} \partial u} & \frac{\partial^{2} J}{\partial T_{s} \partial I_{a k}} \\
\frac{\partial^{2} J}{\partial u \partial T_{s}} & \frac{\partial^{2} J}{\partial u^{2}} & \frac{\partial^{2} J}{\partial u \partial I_{a k}} \\
\frac{\partial^{2} J}{\partial I_{a k} \partial T_{s}} & \frac{\partial^{2} J}{\partial I_{a k} \partial u} & \frac{\partial^{2} J}{\partial I_{a k}^{2}}
\end{array}\right)^{-1}\left(\begin{array}{c}
\frac{\partial^{2} J}{\partial T_{s}} \\
\frac{\partial^{2} J}{\partial u} \\
\frac{\partial^{2} J}{\partial I_{a k}}
\end{array}\right)^{(n)}
\end{aligned}
$$

where

$$
\beta^{(n)}=1 / 2^{n}
$$

In general,

$$
\nabla f(x)=\left(\frac{\partial f(\boldsymbol{x})}{\partial x_{1}}, \frac{\partial f(\boldsymbol{x})}{\partial x_{2}}, \cdots, \frac{\partial f(\boldsymbol{x})}{\partial x_{n}}\right)^{\tau}
$$

and

$$
\nabla^{2} f(x)=\left(\begin{array}{cccc}
\frac{\partial^{2} f(x)}{\partial x_{1}^{2}} & \frac{\partial^{2} f(x)}{\partial x_{1} \partial x_{2}} & \cdots & \frac{\partial^{2} f(x)}{\partial x_{1} \partial x_{n}} \\
\frac{\partial^{2} f(x)}{\partial x_{1} \partial x_{1}} & \frac{\partial^{2} f(x)}{\partial x_{2}^{2}} & \cdots & \frac{\partial^{2} f(x)}{\partial x_{2} \partial x_{n}} \\
\vdots & \vdots & \ddots & \vdots \\
\frac{\partial^{2} f(x)}{\partial x_{n} \partial x_{1}} & \frac{\partial^{2} f(x)}{\partial x_{n} \partial x_{2}} & \cdots & \frac{\partial^{2} f(x)}{\partial x_{n}^{2}}
\end{array}\right)
$$

Therefore, the cost function can be rewritten as follows,

$$
J=f(x), H_{n}=\nabla^{2} f(s)
$$

where

$$
H_{n}=\left(\begin{array}{ccc}
\frac{\partial^{2} J}{\partial T_{s}^{2}} & \frac{\partial^{2} J}{\partial T_{s} \partial u} & \frac{\partial^{2} J}{\partial T_{s} \partial I_{a k}} \\
\frac{\partial^{2} J}{\partial u \partial T_{s}} & \frac{\partial^{2} J}{\partial u^{2}} & \frac{\partial^{2} J}{\partial u \partial I_{a k}} \\
\frac{\partial^{2} J}{\partial I_{a k} \partial T_{s}} & \frac{\partial^{2} J}{\partial I_{a k} \partial u} & \frac{\partial^{2} J}{\partial I_{a k}}
\end{array}\right)
$$

is called Hessian or Hesse matrix.

Equation (1) can be rewritten as follows,

$$
\begin{gathered}
B_{\nu}\left(T_{s}\right)=\frac{c_{i}}{\exp \left(\frac{c_{0} i}{T_{s}}\right)-1} \\
B_{\nu}(T)=\frac{c 1}{\lambda^{3}\left(\exp \left(\frac{c 2}{\lambda T}\right)-1\right)}
\end{gathered}
$$

$$
\begin{aligned}
& c_{i}: 2 h c^{2} / \lambda_{i}^{5} \\
& c_{0 i}: c h / \lambda_{i} k \\
& c 1: 2 h c=1.191126^{-12} \\
& c 2: c h / k=1.43889
\end{aligned}
$$

Then the unknown variables are estimated through iterations. Also Root Mean Square Error: RMSE can be evaluated.

$$
\begin{aligned}
R M S E & =\sqrt{\frac{\sum_{k=1}^{N}\left(e_{k}\right)^{2}}{N}} \\
e_{k} & =T_{s}-T_{s}^{\prime}
\end{aligned}
$$

The first derivatives of the cost function are expressed as follows,

$$
\begin{gathered}
\frac{\partial J}{\partial T_{s}}=-2 \sum_{i=1}^{3}\left(I_{i}-\hat{I}_{i}\right) \frac{\partial \hat{I}}{\partial T_{s}} \\
\frac{\partial J}{\partial u}=-2 \sum_{i=1}^{3}\left(I_{i}-\hat{I}_{i}\right) \frac{\partial \hat{I}}{\partial u} \\
\frac{\partial J}{\partial I_{a k}}=-2 \sum_{i=1}^{3}\left(I_{i}-\hat{I}_{i}\right) \frac{\partial \hat{I}}{\partial I_{a k}}
\end{gathered}
$$

Also the second derivatives are represented as follows,

$$
\begin{aligned}
\frac{\partial^{2} J}{\partial T_{s}^{2}} & =2 \sum_{i=1}^{3}\left\{\frac{\partial \hat{I}_{i}}{\partial T_{s}} \frac{\partial \hat{I}_{i}}{\partial T_{s}}-\left(I_{i}-\hat{I}_{i}\right) \frac{\partial^{2} \hat{I}_{i}}{\partial T_{s}^{2}}\right\} \\
\frac{\partial^{2} J}{\partial T_{s} \partial u} & =2 \sum_{i=1}^{3}\left\{\frac{\partial \hat{I}_{i}}{\partial u} \frac{\partial \hat{I}_{i}}{\partial T_{s}}-\left(I_{i}-\hat{I}_{i}\right) \frac{\partial^{2} \hat{I}_{i}}{\partial T_{s} \partial u}\right\} \\
\frac{\partial^{2} J}{\partial T_{s} \partial I_{a k}} & =2 \sum_{i=1}^{3}\left\{\frac{\partial \hat{I}_{i}}{\partial I_{a k}} \frac{\partial \hat{I}_{i}}{\partial T_{s}}-\left(I_{i}-\hat{I}_{i}\right) \frac{\partial^{2} \hat{I}_{i}}{\partial T_{s} \partial I_{a k}}\right\} \\
\frac{\partial^{2} J}{\partial u \partial T_{s}} & =2 \sum_{i=1}^{3}\left\{\frac{\partial \hat{I}_{i}}{\partial T_{s}} \frac{\partial \hat{I}_{i}}{\partial u}-\left(I_{i}-\hat{I}_{i}\right) \frac{\partial^{2} \hat{I}_{i}}{\partial u \partial T_{s}}\right\} \\
\frac{\partial^{2} J}{\partial u^{2}} & =2 \sum_{i=1}^{3}\left\{\frac{\partial \hat{I}_{i}}{\partial u} \frac{\partial \hat{I}_{i}}{\partial u}-\left(I_{i}-\hat{I}_{i}\right) \frac{\partial^{2} \hat{I}_{i}}{\partial u^{2}}\right\} \\
\frac{\partial^{2} J}{\partial u \partial I_{a k}} & =2 \sum_{i=1}^{3}\left\{\frac{\partial \hat{I}_{i}}{\partial I_{a k}} \frac{\partial \hat{I}_{i}}{\partial u}-\left(I_{i}-\hat{I}_{i}\right) \frac{\partial^{2} \hat{I}_{i}}{\partial u \partial I_{a k}}\right\} \\
\frac{\partial^{2} J}{\partial T_{s} \partial T_{s}} & =2 \sum_{i=1}^{3}\left\{\frac{\partial \hat{I}_{i}}{\partial T_{s}} \frac{\partial \hat{I}_{i}}{\partial I_{a k}}-\left(I_{i}-\hat{I}_{i}\right) \frac{\partial^{2} \hat{I}_{i}}{\partial I_{a k} \partial T_{s}}\right\} \\
\frac{\partial^{2} J}{\partial I_{a k} \partial u} & =2 \sum_{i=1}^{3}\left\{\frac{\partial \hat{I}_{i}}{\partial u} \frac{\partial \hat{I}_{i}}{\partial I_{a k}}-\left(I_{i}-\hat{I}_{i}\right) \frac{\partial^{2} \hat{I}_{i}}{\partial I_{a k} \partial u}\right\} \\
\frac{\partial_{i k}}{\partial I_{a k}} \frac{\partial \hat{I}_{i}}{\partial I_{a k}} & \frac{\partial \hat{I}_{i}}{\partial I_{a k}^{2}}-\left(I_{i}-\hat{I}_{i} \hat{I}_{i}\right.
\end{aligned}
$$

The first derivatives of radiance are expressed as follows,

where 


$$
\begin{gathered}
\frac{\partial I_{i}}{\partial T_{s}}=\frac{c_{i} c_{0 i} c_{1 i}}{T_{s}^{2}\left\{\exp \left(\frac{c_{0 i}}{T_{s}}\right)-1\right\}^{2}} \exp \left\{\frac{c_{0 i}}{T_{s}}-\alpha_{1} u^{\alpha_{2}}\right\} \\
\frac{\partial I_{i}}{\partial u}=c_{1 i} \alpha_{1} \alpha_{2} \alpha_{3} u^{\alpha_{2}-1}\left\{\left(C_{1 i}+C_{2 i} I_{a k}\right)-\frac{c_{i}}{\exp \left(\frac{c_{0 i}}{T_{s}}\right)-1}\right\} \\
\frac{\partial I_{i}}{\partial I_{a k}}=C_{2 i}\left\{1-c_{1 i} \exp \left(-\alpha_{1} u^{\alpha_{2}}\right)\right\}
\end{gathered}
$$

Also the second derivatives of radiance is represented as follows,

$$
\begin{aligned}
& \frac{\partial^{2} I_{i}}{\partial T_{s}}=\left[c_{i} c_{0 i} c_{1 i} \exp \left\{-\alpha_{1} u^{\alpha_{2}}\right\}\right] \\
& \left\{\frac{-c_{0 i} e^{\frac{c_{0 i}}{T_{s}}}-2 T_{s} e^{\frac{c_{0 i}}{T_{s}}}}{T^{4}\left(e^{\frac{c_{01}}{T_{s}}}-1\right)^{2}}+\frac{2 c_{0 i} e^{\frac{c_{0 i}}{T_{s}}}}{T^{4}\left(e^{\frac{c_{01}}{T_{s}}}-1\right)^{3}}\right\} \\
& \frac{\partial^{2} I_{i}}{\partial T_{s} \partial u}=-\frac{c_{i} c_{0 i} c_{1 i}}{T_{s}^{2}\left\{\exp \left(\frac{c_{0 i}}{T_{s}}\right\}^{2}\right.} \exp \left\{\frac{c_{0 i}}{T_{s}}-\alpha_{1} u^{\alpha_{2}}\right\} \alpha_{1} \alpha_{2} u^{\alpha_{2}-1} \\
& \frac{\partial I_{i}}{\partial T_{s} \partial I_{a k}}=0 \\
& \frac{\partial^{2} I_{i}}{\partial u \partial T_{s}}=-\frac{c_{i} c_{0 i} c_{1 i}}{T_{s}^{2}\left\{\exp \left(\frac{c_{0 i}}{T_{s}}\right)-1\right\}^{2}} \alpha_{1} \alpha_{2} \\
& \exp \left\{\frac{c_{0 i}}{T_{s}}-\alpha_{1} u^{\alpha_{2}}\right\} u^{\alpha_{2}-1} \\
& \frac{\partial^{2} I_{i}}{\partial u^{2}}=c_{1 i} \alpha_{1} \alpha_{2}\left\{\left(C_{1 i}+C_{2 i} I_{a k}\right)-\frac{c_{i}}{\exp \left(\frac{c_{0 i}}{T_{s}}\right)-1}\right\} \\
& \frac{\partial^{2} I_{i}}{\partial u \partial I_{a k}}=c_{1 i} C_{2 i} \alpha_{1} \alpha_{2} \alpha_{3} u^{\alpha_{2}-1} \\
& \frac{\partial^{2} I_{i}}{\partial I_{a k} \partial T_{s}}=0 \\
& \frac{\partial^{2} I_{i}}{\partial I_{a k} \partial u}=c_{1 i} C_{2 i} \alpha_{1} \alpha_{2} \alpha_{3} u^{\alpha_{2}-1} \\
& \frac{\partial^{2} I_{i}}{\partial I_{a k}^{2}}=0
\end{aligned}
$$

where

$$
\begin{gathered}
\alpha_{1}=c_{2 i}+\frac{c_{3 i}}{\cos \theta} \\
\alpha_{2}=c_{4 i}+\frac{c_{5 i}}{\cos \theta} \\
\alpha_{3}=\exp \left\{-\alpha_{1} u^{\alpha_{2}}\right\}
\end{gathered}
$$

\section{E. Assumed Spectral Bands}

Spectral bands of ADEOS/OCTS (Advanced Earth
Observing Satellite / Ocean Color and Temperature Scanner) are assumed as typical spectral bands for SST estimation which are 10300-11360nm for Band 4, and 11360-12500nm for Band 5 , respectively.

\section{EXPERIMENTS}

\section{F. Simulation Conditions}

The following parameters are set for the experiments with MODTRAN obtaining at sensor radiance of spectral TIR band data.

\author{
Atmospheric Model: Tropic, Mid.Latitude Summer, \\ Mid.Latitude Winter, SubArctic Summer, SubArctic \\ Winter, 1976 US Standard Atmosphere \\ Constraint: $\pm 2[\mathrm{~K}]$ \\ Meteorological Range: $\pm 0 \%, \pm 10 \%, \pm 20 \%$ \\ Relative Humidity: Default x1.0,x.1.1,x1.2,x0.9,x0.8 \\ Air-Temperature: Default $\pm 0, \pm 3[\mathrm{~K}]$ \\ Sea surface temperature: Default $\pm 0, \pm 3[\mathrm{~K}]$ \\ Wind speed: $3.5,7.0,14.0[\mathrm{~m} / \mathrm{s}]$
}

Aerosol Model: Navy Maritim,Maritim,Tropospheric,Desert

Observation Zenith Angle: $0,30,60[\mathrm{deg}]$

Then SST is estimated with the proposed method and the conventional split window method using the calculated at sensor radiance.

\section{G. Evaluation of RMSE for the Proposed CGM}

SST estimation error for the proposed CGM can be evaluated with RMSE which is expressed in equation (23) by using MODTRAN derived at sensor radiance of TIR bands.

Figure 1 show RMSE of CGM as functions of (a) relative humidity, (b) meteorological range, (c) air temperature, (d) observation zenith angle, (e) sea surface temperature, and (f) wind speed for six atmospheric models. In accordance with increasing of relative humidity, meteorological range, observation zenith angle, sea surface temperature, and wind speed, RMSE increased. RMSE is, on the other hands, decreases in accordance with increasing of air temperature. Meanwhile, Figure 2 (a) shows the relation between meteorological range and RMSE as parameters of different types of aerosol for the Mid.

Latitude Summer of atmospheric model. RMSE for Navy Maritime shows the greatest followed by Maritime, Desert, and Troposphere aerosol. Figure 2 (b) shows RMSE for the six different aerosol types, Navy Maritime, Maritime, Urban, Desert, Rural, and Troposphere aerosol types at the meteorological range of $23 \mathrm{~km}$. Figure 2 (c) shows RMSE as function of altitude for Desert aerosol. Figure 3 shows RMSE of the representative radiance from the atmosphere for the six different atmospheric models. It does not show monotonic relation between relative humidity and RMSE. Therefore, the representative radiance from the atmosphere has to be estimated precisely. 


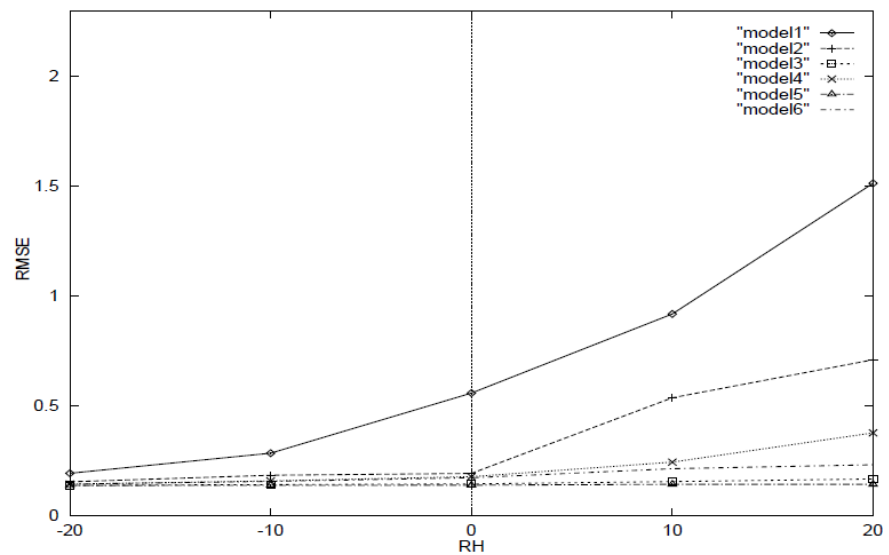

(a)Relative Humidity

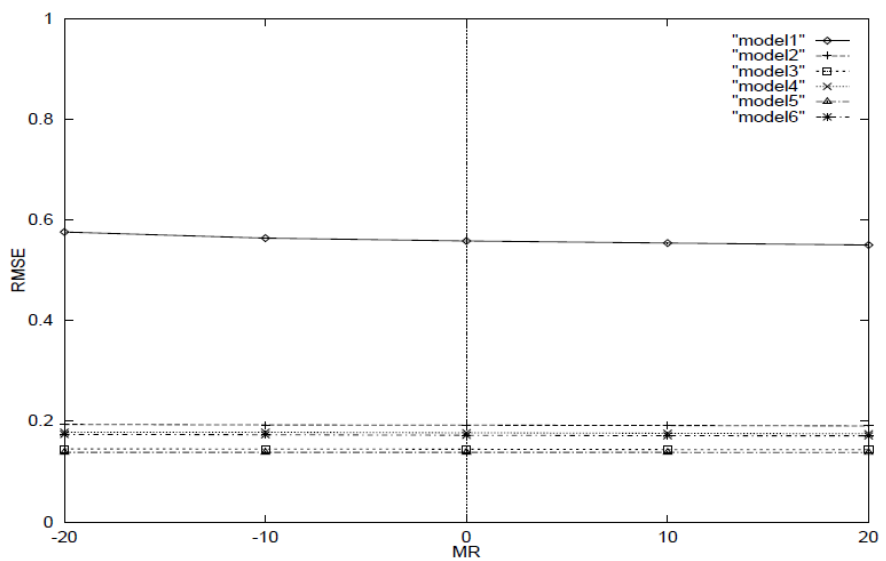

(b)Meteorological Range

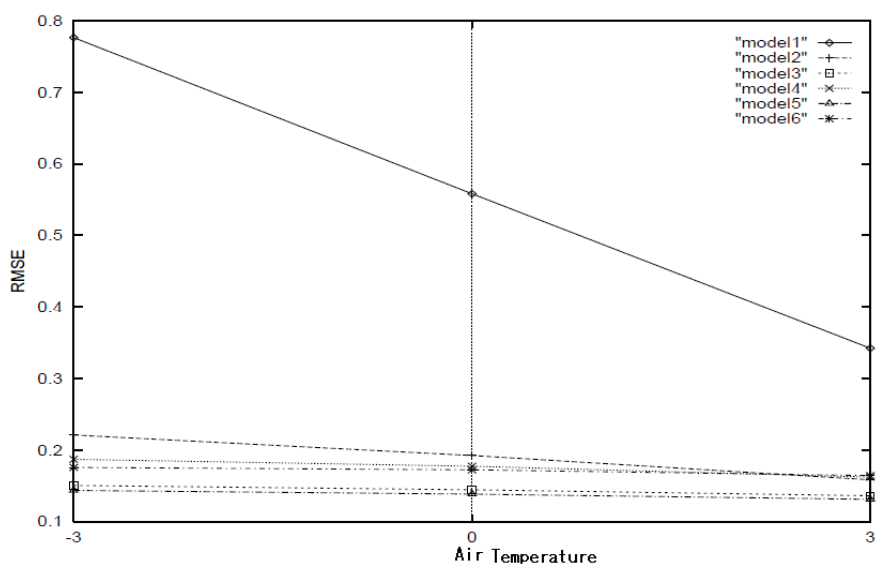

(c)Air Temperature

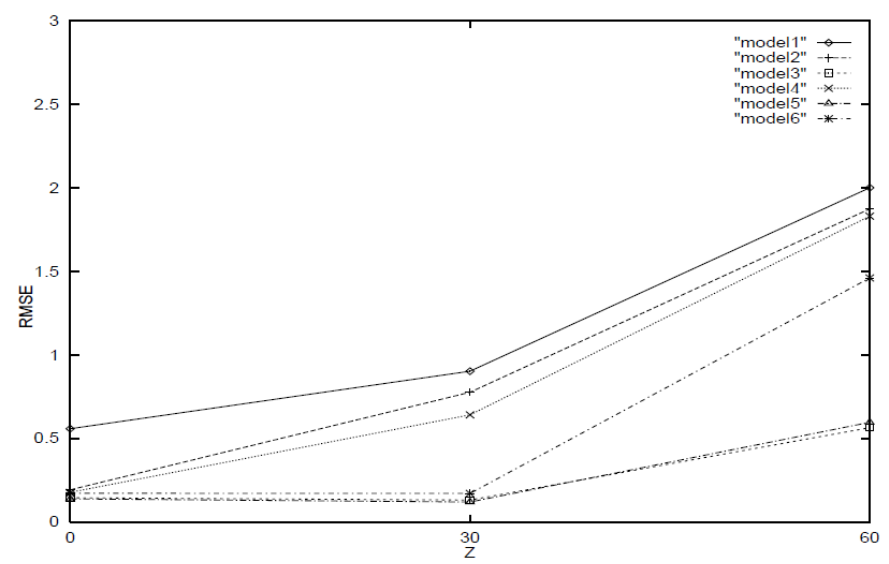

(d)Observation Zenith Angle

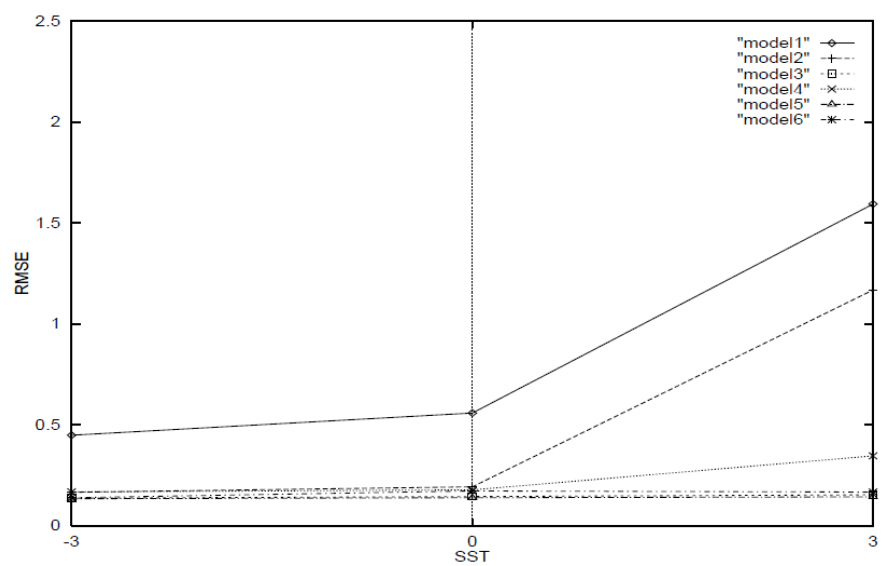

(e)Sea Surface Temperature

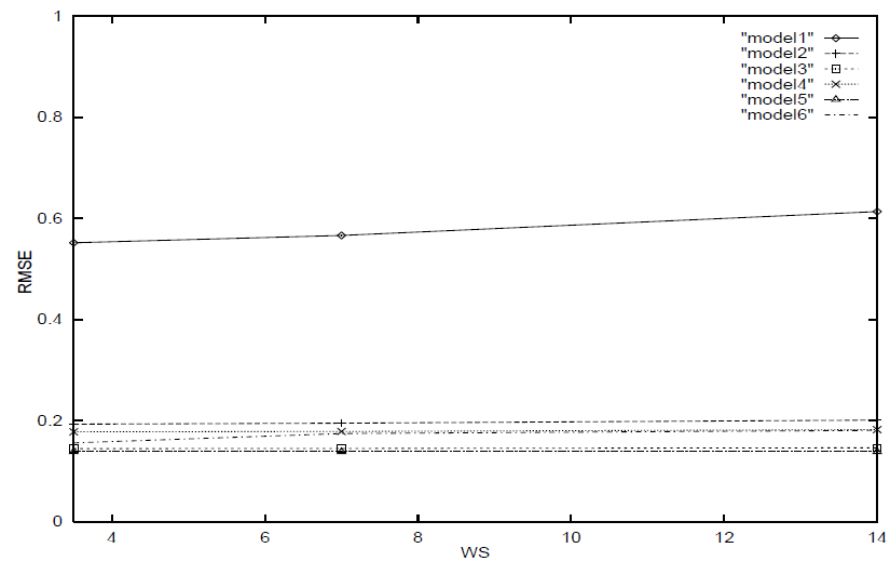

(f)Wind Speed

Fig. 1. RMSE of CGM as functions of (a) relative humidity, (b) meteorological range, (c) air temperature, (d) observation zenith angle, (e) sea surface temperature, and (f) wind speed for six atmospheric models 


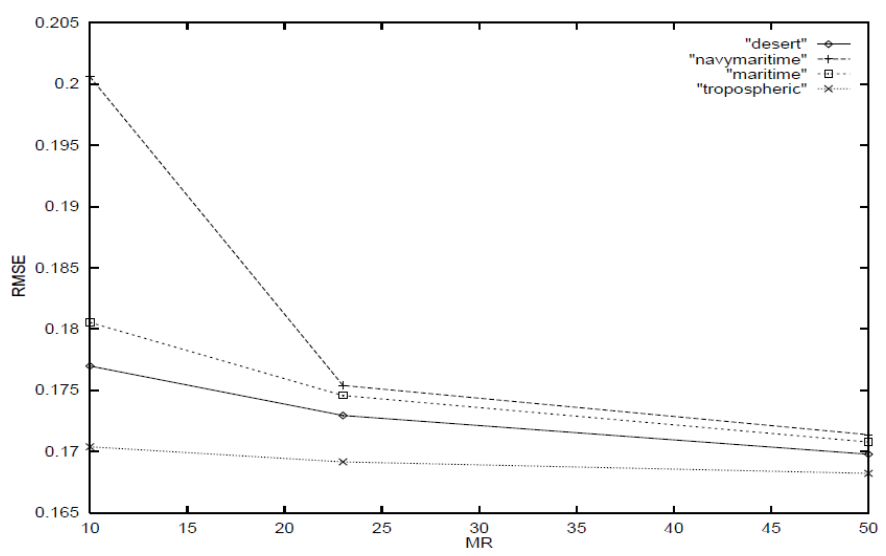

(a)Aerosol Type

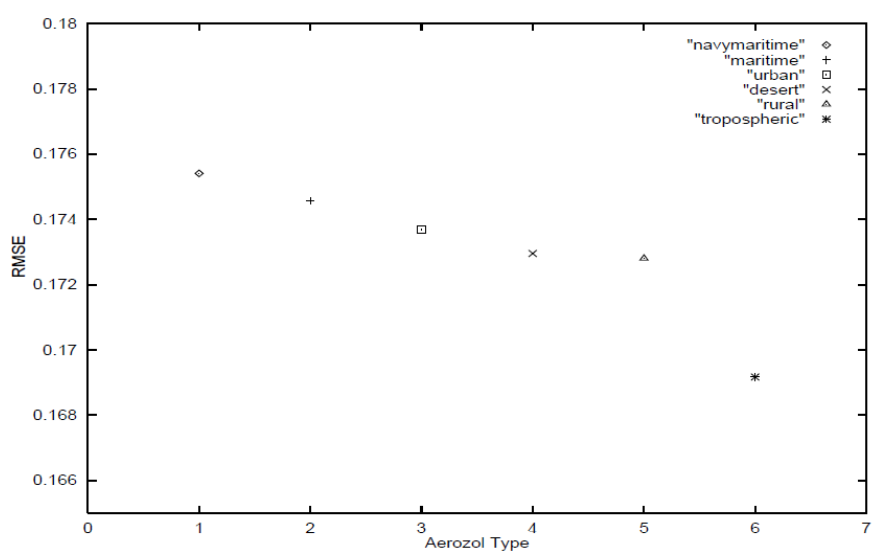

(b)Aerosol Type

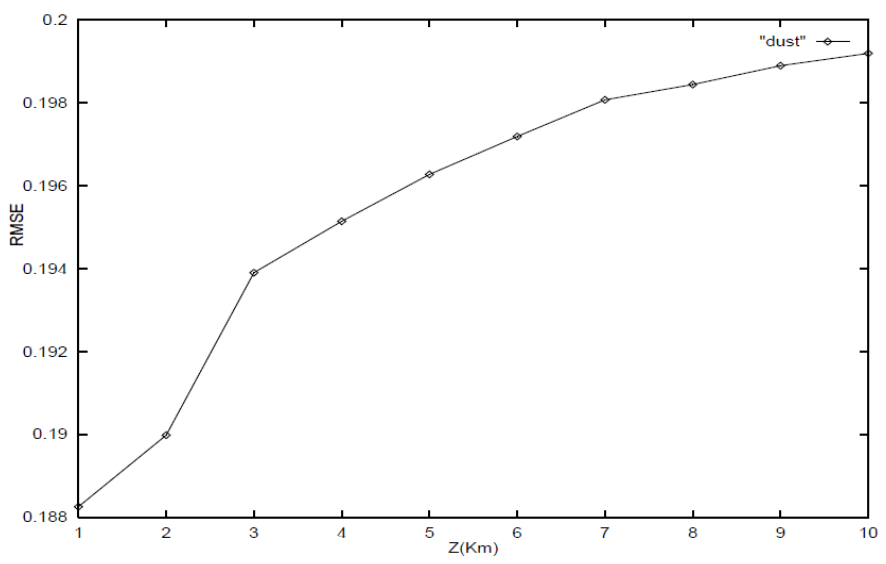

(c)Altitude (Desert type of aerosol)

Fig. 2. RMSE as function of aerosol types

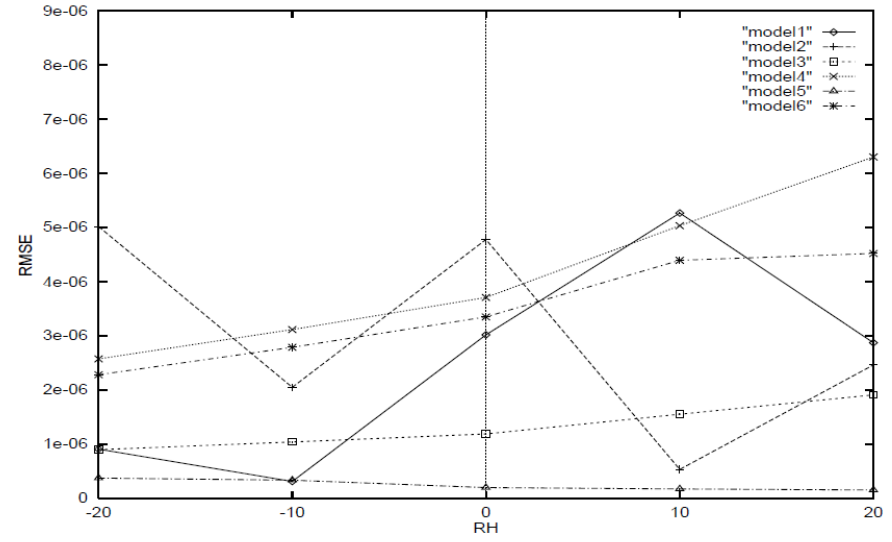

Fig. 3. RMSE of the representative radiance from the atmosphere for the six different atmospheric models

\section{H. Evaluation of RMSE for the Conventional Split Window}

SST estimation error for the conventional split window can be evaluated with RMSE which is expressed in equation (23) by using MODTRAN derived at sensor radiance of TIR bands. Figure 4 shows RMSE of Split Window as functions of (a) relative humidity, (b) meteorological range, (c) air temperature, (d) observation zenith angle, (e) sea surface temperature, and (f) wind speed for six atmospheric models. RMSE of the conventional Split Window is much larger than that of CGM. In accordance with increasing of relative humidity, meteorological range, observation zenith angle, sea surface temperature, and wind speed, RMSE increased. RMSE is, on the other hands, decreases in accordance with increasing of air temperature.

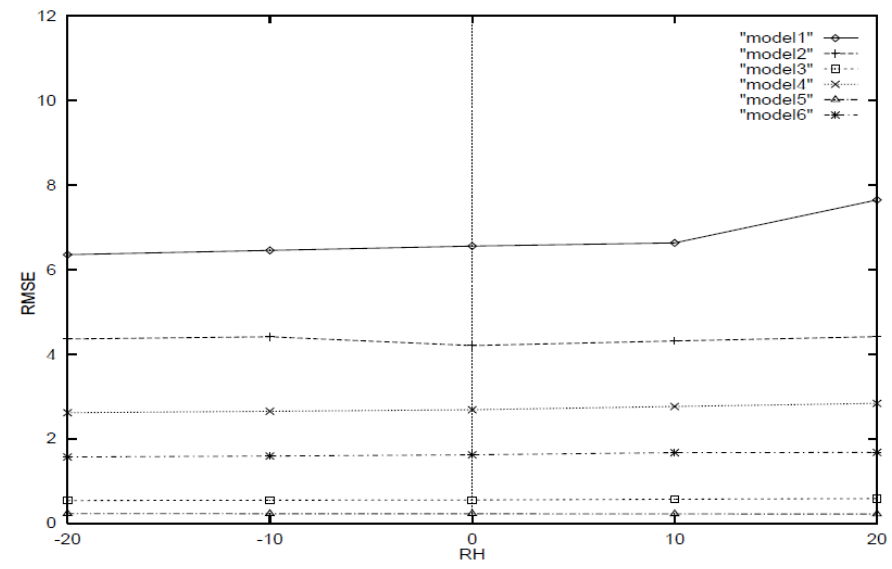

(a)Relative Humidity 


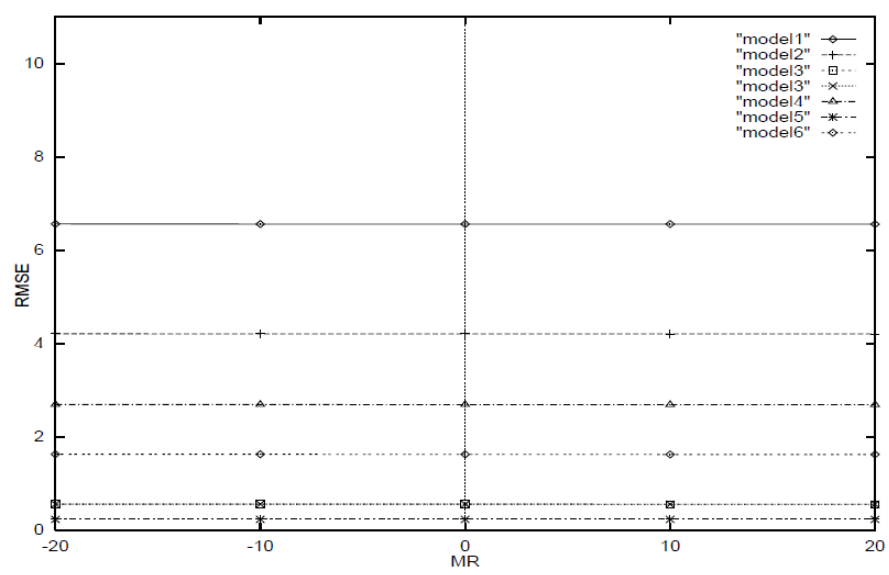

(b)Meteorological Range

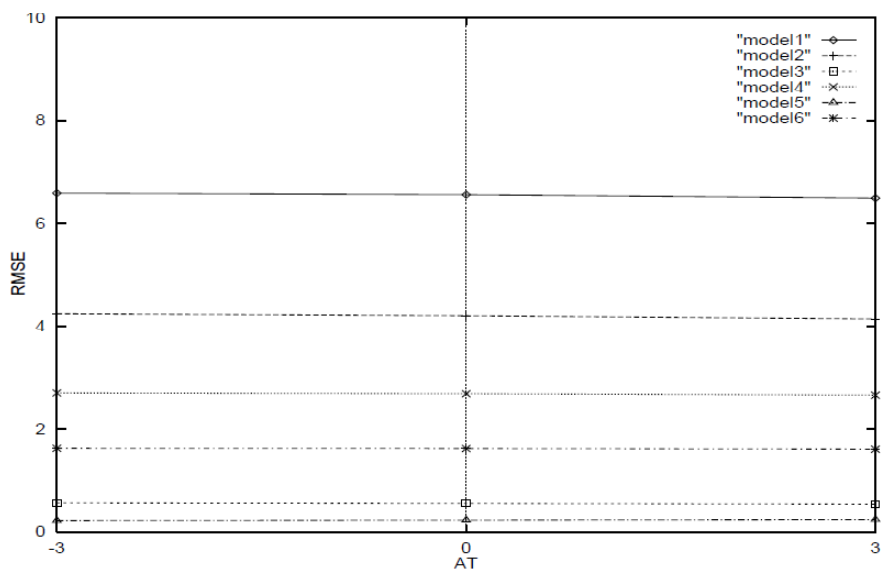

(c)Air Temperature

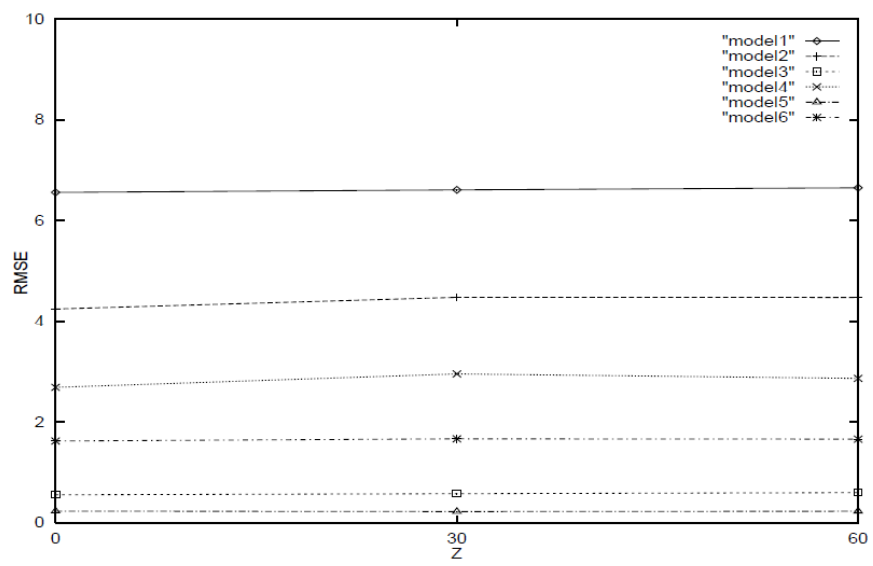

(d)Observation Zenith Angle

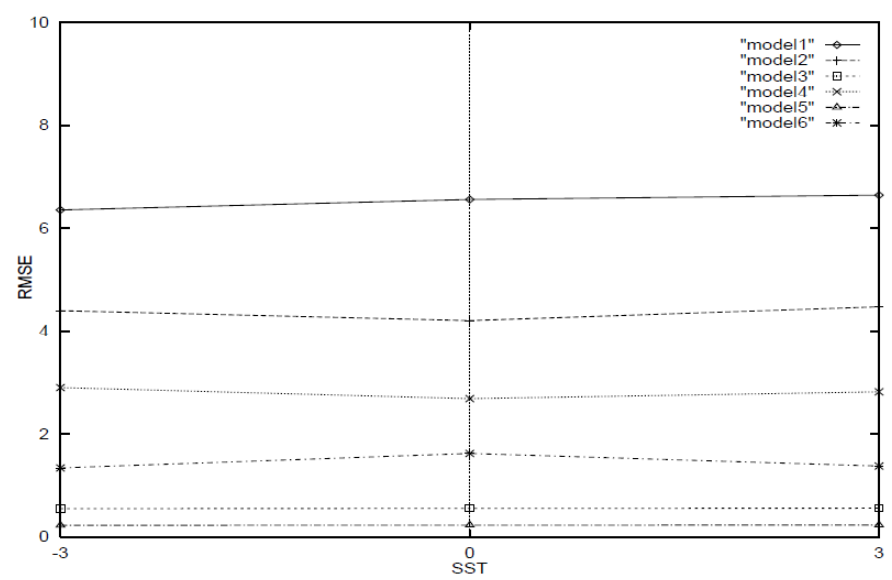

(e)Sea Surface Temperature

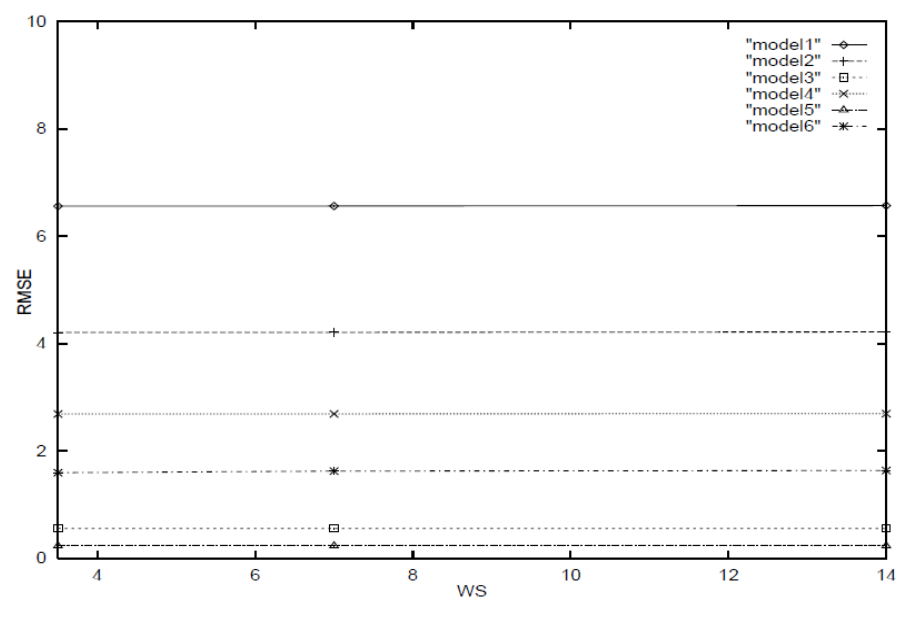

(f)Wind Speed

Fig. 4. RMSE of the conventional Split Window as functions of (a) relative humidity, (b) meteorological range, (c) air temperature, (d) observation zenith angle, (e) sea surface temperature, and (f) wind speed for six atmospheric models

\section{Comparison of RMSE between Split Window and CGM}

Overall RMSE of the conventional Split Window and the proposed CGM is shown in Table 3.

Although depending on the atmospheric model, RMSE between both are different, RMSE of the proposed CGM is lower than that of Split Window. Therefore, CGM is superior to Split Window. 
TABLE III. OVERALL RMSE OF THE CONVENTIONAL SPLIT WINDOW AND THE PROPOSED CGM

\begin{tabular}{|c|r|r|}
\hline & Split Window & Conjugate Gradient \\
\hline Tropic & 1.195 & 0.864 \\
\hline Mid. Latitude Summer & 0.702 & 0.479 \\
\hline Mid. Latitude Winter & 0.472 & 0.408 \\
\hline Sub Arctic Summer & 0.641 & 0.596 \\
\hline Sub Arctic Winter & 0.483 & 0.317 \\
\hline 1976 US Standard & 0.505 & 0.478 \\
\hline Average & 0.726 & 0.559 \\
\hline
\end{tabular}

\section{J. Influence Due to Observation Noise}

In order to evaluate influence due to observation noise on SST estimation accuracy, RMSE with and without of random number derived noise is evaluated. The normal distribution of random number with $10^{-6}$ of variance and with zero mean is generated by using Messene Twister. The random number is added to the at sensor radiance of the simulated TIR bands data. Then SST is estimated based on the proposed conjugate gradient method. Table 4 shows the result.

TABLE IV. INFLUENCE DUE TO OBSERVATION NOISE ON SST ESTIMATION ACCURACY

\begin{tabular}{|c|r|r|}
\hline & Without noise & With $10^{\wedge}-6$ of noise \\
\hline Tropic & 0.4386 & 0.7214 \\
\hline Mid. Latitude Summer & 0.1924 & 0.6012 \\
\hline Mid. Latitude Winter & 0.1446 & 0.2871 \\
\hline Sub Arctic Summer & 0.1774 & 0.5624 \\
\hline Sub Arctic Winter & 0.1384 & 0.2601 \\
\hline 1976 US Standard & 0.1724 & 0.3631 \\
\hline
\end{tabular}

\section{IV. CONCLUSION}

Sensitivity analysis on Sea Surface Temperature: SST estimation with Thermal Infrared Radiometer: TIR data through simulations is conducted. Also Conjugate Gradient Method: CGM based SST estimation method is proposed. SST estimation error of the proposed CGM based method is compared to the conventional Split Window Method: SWM with a variety of conditions including atmospheric models. The results show that the proposed CGM based method is superior to the SWM.

\section{ACKNOWLEDGMENT}

The author would like to thank Mr. Noriyuki Takamatsu for his contributions to conduct experiments.

\section{REFERENCES}

[1] Anding, D. and R. Kauth (1970): Estimation of sea surface temperature from space. Remote Sens. Environ., 1, 217-220.

[2] McMillin, L. M. (1975): Estimation of sea surface temperatures from two infrared window measurements with different absorption. $J$. Geophys. Res., 80, 5113-5117.

[3] Prabhakara, C., G. Dalu and V. G. Kunde (1974): Estimation of sea surface temperature from remote sensing in the 11- to 13- $\mu \mathrm{m}$ window region. J. Geophys. Res., 79, 5039-5044.

[4] Kohei Arai, Preliminary Assessment of Radiometric Accuracy for MOS1 Sensors, International Journal of Remote Sensing, Vol.9, No.1, pp.512, Apr.1988.

[5] Kidwell, K. B. (1991): NOAA Polar Orbiter Data Users Guide. NOAA/NESDIS/NCDC/SDSD, Washington, D.C., 192 pp.

[6] Barton, I. J. (1995): Satellite-derived sea surface temperatures: Current status. J. Geophys. Res., 100, 8777-8790.

[7] Moriyama,M., K.Mima and K.Arai, Comparison of the sea surface temperature estimation methods, Advances in Space Reserach, Vol.16, NO.10, pp.121-124, A1-044, July 1994.

[8] K.Arai, Hidemasa Kobayashi, Masao Moriyama, Sea surface temperature estimation method based on Inversion and Linearized Inversion, Journal of Remote Sensing Society of Japan, 18, 3, 43-50, 1998.

[9] K.Arai, Optimum band selection for sea surface teperature estimation based on regressive analysis, Journal of Japanese Remote Sensing and Photogrammetry, 39, 2, 74-81, 2000.

[10] K.Arai, Sea Surface Temperature estimation method for ocean areas and seasons using Geographic Information System as a neural network, Sediment and Ecohydraulics, Proc.in Marine Science, 9, Elsevier ISBN978-0-444 53184-1, 2007

\section{AUTHORS PROFILE}

Kohei Aarai He received BS, MS and PhD degrees in 1972, 1974 and 1982 respectively. He was with The Institute for Industrial Science and Technology of the University of Tokyo from April 1974 to December 1978 and also was with National Space Development Agency of Japan from January, 1979 to March, 1990. During from 1985 to 1987, he was with Canada Centre for Remote Sensing as a Post Doctoral Fellow of National Science and Engineering Research Council of Canada. He moved to Saga University as a Professor in Department of Information Science on April 1990. He was a councilor for the Aeronautics and Space related to the Technology Committee of the Ministry of Science and Technology during from 1998 to 2000. He was a councilor of Saga University for 2002 and 2003. He also was an executive councilor for the Remote Sensing Society of Japan for 2003 to 2005. He is an Adjunct Professor of University of Arizona, USA since 1998. He also is Vice Chairman of the Commission-A of ICSU/COSPAR since 2008. He wrote 30 books and published 332 journal papers. 